\title{
Cascade Forward Artificial Neural Network based Behavioral Predicting Approach for the Integrated Satellite-terrestrial Networks
}

\author{
Mingchuan Yang ${ }^{1,2}\left[\right.$ (D) Bingyu Xie ${ }^{1} \cdot{\text { Yingzhe } \text { Dou }^{1} \cdot \text { Guanchang Xue }}^{1}$
}

Accepted: 27 September 2021 / Published online: 5 February 2022

(c) The Author(s) 2021

\begin{abstract}
In order to reduce the risk of authorized users being interrupted in the cognitive satellite wireless network, a multi-step prediction approach based on a cascaded forward artificial neural network is proposed to predict user behavior in the designed scenario. This approach uses the powerful learning ability of the cascaded forward network to analyze the historical spectrum occupancy records of licensed users, and then predict the user behavior in the next few time slots. The prediction result can help the base station in the cognitive network to schedule the dynamic access process of the cognitive users, and reduce the interference caused by the cognitive user to the authorized users. Finally, compared with traditional prediction algorithms, it is verified that the proposed multi-step prediction algorithm can effectively reduce the probability of spectrum conflicts.
\end{abstract}

Keywords Behavior prediction $\cdot$ Dynamic spectrum access $\cdot$ Spectrum conflict $\cdot$ Satellite network

\section{Introduction}

In recent years, with the advantages of large coverage and long communication distance, satellite communication technology has been widely used in global business, education and entertainment [1]. High data rates and ubiquitous services result in a large amount of energy consumption for transmitters and receivers. At the same time, the demand for fixed and mobile satellite broadband access is also growing. In order to improve the transmission efficiency of satellite communication, people put forward the idea of integrated satellite terrestrial networks [2], that is, both satellite and terrestrial network work in the S-band to save the cost of facilities and spectrum resources. In addition, the signal fading of higher frequency bands (such as Ka band) in satellite communication cannot be ignored [4]. Therefore, in the satellite communication system, the growing demand for high-speed multimedia services and the transmission characteristics of signals put forward challenges to the limited spectrum resources. More efficient and reasonable utilization

Mingchuan Yang

mcyang@hit.edu.cn

1 Communication Research Center, Harbin Institute of Technology, Harbin, China

2 Science and Technology On Communication Networks Laboratory, Shijiazhuang 050002, China of limited spectrum resources in satellite communication systems has gradually become a hot issue.

Although frequency resources are becoming more and more precious, the spectrum utilization rate of satellite communication systems is low [5]. The frequency band occupied by most fixed satellite communication systems is above $4 \mathrm{GHz}$, but the spectrum allocation strategy is unreasonable, and the test results show that the spectrum utilization rate is very low [6]. The dynamic spectrum technology based on cognitive radio can effectively solve the problem of spectrum resource utilization, which has been studied by many scholars [7]. In 2003, dynamic spectrum allocation technology based on cognitive radio is the main research direction of "next generation program" (XG) proposed by Defense Advanced Research Projects Agency [8]. Reference [9] describes the statistical interference model of terrestrial wireless communication system to satellite communication system in the satellite cognitive radio network, and evaluates the interference between two coexisting systems. Reference[10] proposed a multi beam satellite cognitive system based on beam hopping, which can significantly improve the spectrum utilization of satellite communication system. Reference [11] analyzes the future development prospects of cognitive wireless network technology in satellite communications, and points out the new application of cognitive wireless network technology and multimedia-related technologies in satellite communications. 
The problem of low spectrum utilization has become a prerequisite for the introduction of cognitive radio technology. However, satellite communication systems have high requirements for the stability of signal transmission, and interference from cognitive users to authorized users should be avoided [12]. In satellite cognitive wireless networks, the signal transmission delay will cause the time lag for cognitive users to sensing authorized users. Therefore, the focus is on the behavior prediction technology of authorized users in the satellite cognitive radio network, the artificial neural network is used for behavior prediction, and a multi-step prediction algorithm based on the cascaded forward neural network is proposed.

The paper is organized as follows. In Sect. 2, we introduce the behavior prediction model of the satellite cognitive radio networks. In Sect. 3, the user behavior prediction algorithms are investigated, and a new multi-step behavior predicting algorithm based on cascade forward artificial neural network is proposed. The performance of the behavior prediction algorithms is verified in Sect. 4. Finally, the conclusion is provided in Sect. $5 \mathrm{~s}$.

\section{Behavior Prediction Model}

\subsection{Behavior Prediction System Model}

Assume that the cognitive network sensing the bandwidth $B$ of the frequency band, where $f_{\max }$ and $f_{\min }$ are the upper and lower boundaries of the target frequency band, respectively. According to the transmission performance requirements of cognitive users, the target frequency band is divided into $N$ sub-bands with bandwidth $B$, and $B \times N \leq W$. Therefore, the cognitive network base station can obtain the sensing results of $N$ sub-bands in each sensing process. If the authorized user is occupying the sub-band, then $\mathrm{s}_{i}=1(i=1,2, \ldots, N)$, otherwise $\mathrm{s}_{i}=0$. In addition, assume that the length of the sensing time slot of the cognitive network is $\Delta t$. The process of establishing a spectrum database by a cognitive base station is shown in Fig. 1. The process of establishing a spectrum database by a cognitive base station needs to weigh the processing complexity, the effectiveness of spectrum resource allocation, the length of the sub-band sensing time slot $\Delta$ tand the bandwidth $B$.

In the dynamic spectrum access scenario of the satellite cognitive radio network, the cognitive base station uses the information sensed by the sensing node to obtain the spectrum occupancy of the sub-band, and stores it in the spectrum database. Then the user behavior prediction system

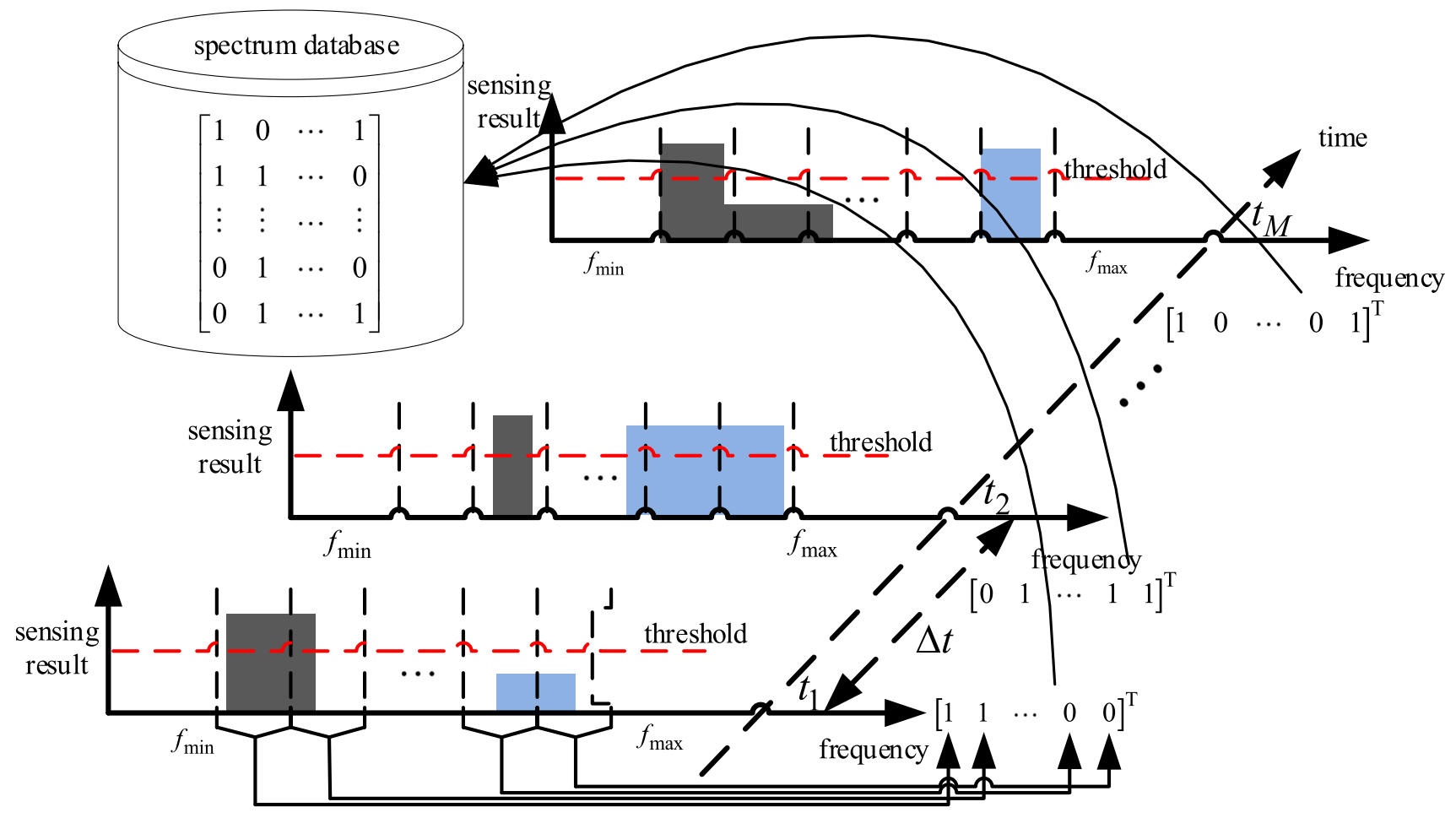

Fig. 1 Cognitive base station spectrum database formation process 
uses the established spectrum database and the behavior of authorized users at the current time to predict the behavior in the next several time slots. The prediction system performs information extraction and self-learning based on the historical behavior of authorized users. The ground area covered by non-geostationary orbit satellites is periodic, and the data flow of geostationary orbit satellites also has a certain distribution law [13]. The user behavior prediction system uses the distribution law of authorized users' spectrum occupancy behavior in the time dimension as a prediction basis. In order to reduce the prediction time and improve the realtime performance of the prediction results, the prediction system needs to learn and extract features in advance, and use the current spectrum sensing results to make predictions. Therefore, for the non-geostationary satellites in the satellite cognitive radio network, the ground-based cognitive network can use the time when the satellite is not visible for feature extraction. For the geostationary satellites, the ground-based cognitive network can only use the historical information in the spectrum database.

\subsection{User Behavior Model}

The behavior of authorized users in cognitive radio networks is not completely random, but is affected by human activities and follows certain mathematical models [14]. Therefore, cognitive users can predict the behavior of authorized users. In traditional work, modeling the behavior of authorized users with deterministic traffic is relatively mature, while random traffic modeling usually adopts exponential Poisson distribution model and Pareto distribution model [[[15-17]]]. In this paper, the exponential ON/OFF model is used to model the behavior of authorized users, and on this basis, the behavior prediction algorithm is studied.

The behavior of authorized users using any sub-band $i$ $(i=1,2, \ldots, N)$ can be represented by a time continuous semiMarkov process [16]. For the sub-band $i$, the ON model indicate the occupied state $T_{O N}^{i}$ and the OFF model indicate the unoccupied state $T_{O F F}^{i}$ Therefore, the state of each sub-band is constantly changing between $\mathrm{ON}$ and $\mathrm{OFF}$, and the cognitive users' sensing results can be represented by a sequence of 1 and 0 accordingly. Assumed that $T_{O N}^{i}$ and $T_{O F F}^{i}$ are described by independent and identically distributed random variables $Y^{i}$ and $X^{i}$ respectively.

Firstly, we assume that $T_{O N}^{i}$ obeys the exponential distribution with the expectation of $e_{1}=\frac{1}{\lambda_{1}}$ :

$T_{O N}^{i} \sim f_{Y_{i}}(y)=\left\{\begin{array}{cl}\lambda_{1} e^{-\lambda_{1} y} & , y \geq 0 \\ 0 & , y<0\end{array}\right.$

Similarly, suppose that $T_{\rho F F}^{i}$ obeys exponential distribution with expectation $e_{2}=\frac{\rho F F}{\lambda_{2}}$, that is:
$T_{O F F}^{i} \sim f_{Y_{i}}(x)=\left\{\begin{array}{cc}\lambda_{1} e^{-\lambda_{2} y} & , y \geq 0 \\ 0 & , y<0\end{array}\right.$

The value $e_{1}$ and $e_{2}$ are the service parameter of authorized user, which respectively reflect the average time of subband in the ON and OFF state. The greater the value $T_{O N}^{i}$ and $T_{O F F}^{i}$, the longer the authorized user stays in a certain state, and vice versa, the higher the frequency of state transitions. Therefore, the process of predicting the behavior of authorized users is to use the sensing results of the binary sequence at the current moment and the past moment to estimate the value of the sequence in the next few time slots.

\section{User behavior Prediction Algorithm}

\subsection{User Behavior Prediction Algorithm Based on Update Theory}

For the exponential ON/OFF model, the update theory [18] can be used to predict the behavior of authorized user. The prediction algorithm based on update theory uses the current state of sensing result to predict the behavior in the next time slot. For sub-band $i(i=1,2, \ldots, N)$, assuming that its state in the current sensing slot is $s_{i}, s_{i} \in(\mathrm{ON}, \mathrm{OFF})$. In the next sensing time slot, the probability that the sub-band is not occupied is $P_{i}$.

$P_{i}=\left\{\begin{array}{l}\frac{\lambda_{1}+\lambda_{2} \cdot e^{-\left(\lambda_{1}+\lambda_{2}\right) \Delta t}}{\lambda_{1}+\lambda_{2}}, S_{i}=O N \\ \frac{\lambda_{1}+\lambda_{2} \cdot e^{-\left(\lambda_{1}+\lambda_{2}\right) \Delta t}}{\lambda_{1}+\lambda_{2}}, S_{i}=O F F\end{array}\right.$

However, the shortcoming of this algorithm is also obvious. In order to predict user behavior, the cognitive base station must know the ON/OFF model parameters of the user behavior in advance, that is, the value of $\lambda_{1}$ and $\lambda_{2}$ must be obtained in advance. This is very difficult for base stations in satellite cognitive wireless networks, because authorized users are not obligated to inform cognitive users of their behavior models, and the authorized users 'model may change over time. In actual situations, cognitive base stations can only make predictions based on historical information in the spectrum database.

\subsection{One Step Prediction Algorithm Based on Feed-forward Neural Network (FFN)}

In the cognitive wireless network, the cognitive base station usually does not know the law of authorized users to occupy the spectrum, and can only predict it through the binary sequence stored in the spectrum database. The onestep prediction algorithm based on the feedforward neural 
network is based on this idea [19], and uses the powerful nonlinear mapping ability of the feedforward artificial neural network to use the binary sequence of the current and previous moments as the input of the network. After training and learning under a certain number of samples, the feedforward network can predict state in the next time slot.

Assume that the binary sequence $S=\left\{s_{1}, s_{2}, \ldots s_{t}\right\}, s_{i} \in$ $\{0,1\}$ represents the history data stored in the spectrum database, and $s_{t}$ is the sensing result at current moment. FNN takes the binary data $\left[S_{t-\tau+1}, \mathrm{~S}_{t-\tau+2}, \ldots S_{t-1}, S_{t}\right]^{T}$ with the time window of length $\tau$ as input, and the output $\widehat{s}_{t+1}$ is the predicted state in the next time slot. The time window length $\tau$ is also called prediction order. FFN uses a sliding time window to continuously predict user behavior. As time goes by, when FFN obtains the $S_{t+1}$ at the next moment, it will be used to update the time window, that is, the neural network uses $\left[S_{t-\tau+2}, S_{t-\tau+3}, \ldots S_{t}, S_{t+1}\right]^{T}$ as input to predict $S_{t+2}$.

Before using FFN to predict user behavior, the network needs to be trained first. The authorized user status information in the spectrum database is divided into training samples, and each group of training samples includes an input of length $\tau$ and an expected output $S_{t+2}$ FFN continuously adjusts the network parameters through the error back propagation (BP) algorithm to narrow the gap $e_{t}$ between its output $\widehat{s}_{t+1}$ and the expected value $S_{t+1}$.

Artificial neural network is the realization of user feature extraction and nonlinear mapping in the prediction process. It is a massively parallel computing system connected by a large number of simple processors, which realizes information processing through the interaction between neurons. The basic artificial neural network consists of at least three layers, namely an input layer, an output layer and at least one hidden layer. The front and back layers are fully connected, and there is no connection between neurons in each layer.
The model of an artificial neural network with a hidden layer is shown in Fig. 2. The input layer has $I$ neurons, the output layer has $K$ neurons, and the hidden layer has $J$ neurons, $w_{j i}^{J I}(j=1,2, \ldots J ; i=1,2, \ldots, I)$ represents the weight between the input layer and the hidden layer. $b_{j}^{J}(j=1,2, \ldots, J)$ and $b_{k}^{K}(k=1,2, \ldots, K)$ represent the bias of the hidden layer and the output layer, respectively.

Each input layer neuron is connected to the hidden layer, and the input signal is assigned to the hidden layer through a weight matrix. Any node $j$ in the hidden layer performs a summation operation on the input signal and adds the offset $b_{j}^{J}$ to form the net input $v_{j}^{J}$ of the node, which is then processed with a specific transfer function. Usually the hyperbolic tangent $\mathrm{S}$ function $\varphi(\bullet)$ is used as the transfer function, then the output $o_{j}^{J}$ of the $j$-th hidden layer neuron can be expressed as:

$o_{j}^{J}=\varphi v_{j}^{J}=\frac{1-\exp \left(-v_{j}^{J}\right)}{1+\exp \left(-v_{j}^{J}\right)}$

where $v_{j}^{J}=\sum_{i=1}^{I} w_{j i}^{J I} o_{j}^{I}+b_{j}^{J}$, and $o_{j}^{I}$ is the output of $i$-th neuron in the input layer. Then $o_{j}^{I}(j=1,2, \ldots, J)$ undergoes the same process in the output layer, the final output signal $o_{k}^{K}=\varphi\left(v_{k}^{K}\right),(k=1,2, \ldots, K)$ of the neural network can be obtained.

In order to make the actual output $o_{k}^{K}$ close to the expected value $d_{k}$, the weight of the network needs to be adjusted through the BP algorithm. Define the cost function $\varepsilon$ as the energy of the error between the actual output and the expected value.
Fig. 2 Artificial Neural Network Model

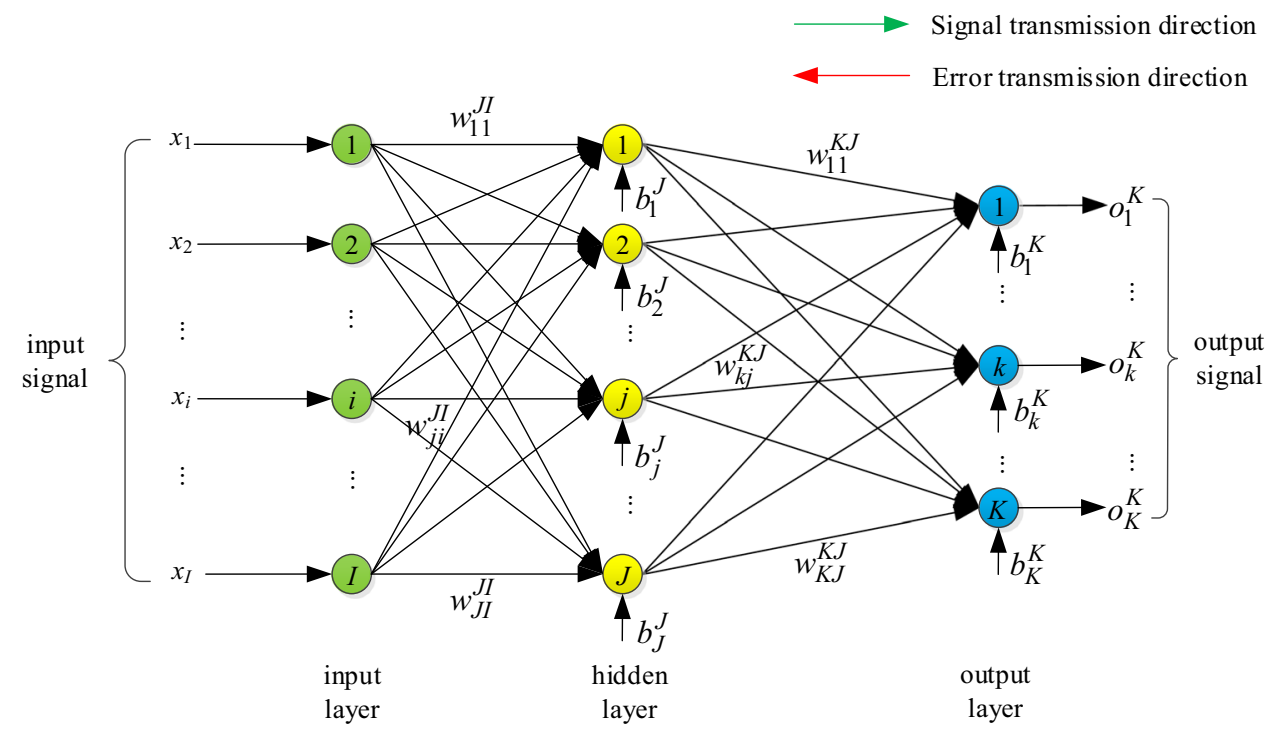


$\varepsilon=E=\frac{1}{2}\left[\sum_{k=1}^{K}\left(d_{k}-o_{k}^{K}\right)^{2}\right]$

The neural network gradually reduces the cost function $\varepsilon$ by adjusting the weight of each layer. The BP learning algorithm uses a gradient descent method to modify the weights layer by layer through continuous iteration. For the output layer, the process of BP algorithm to modify its weight $v_{k j}^{K J}$ is as follows:

$w_{k j}^{K J}(n+1)=w_{k j}^{K J}(n)+\Delta w_{k j}^{K J}$

where $w_{k j}^{K J}(n)$ is the weight of the layer at the $n$-th iteration. $\Delta w_{k j}^{K J}$ is the correction amount of the weight, which satisfies:

$\Delta w_{k j}^{K J}=\eta_{k}\left(-\frac{\partial \varepsilon}{\partial w_{k j}^{K J}}\right)=\eta_{k}\left(-\frac{\partial \varepsilon}{\partial v_{k}^{K}}\right) \times\left(\frac{\partial v_{k}^{K}}{\partial w_{k j}^{K J}}\right)$

where $\eta_{k}$ is the learning rate of the $k$-th neuron, and the net input of this neuron is $v_{k}^{K}=\sum_{j=1}^{J} w_{k j}^{K J} o_{j}^{J}+b_{k}^{K}$, then we have:

$\left(\frac{\partial v_{k}^{K}}{\partial w_{k j}^{K J}}\right)=o_{j}^{J}$

The term $\left(-\frac{\partial \varepsilon}{\partial \nu_{k}^{K}}\right)$ in Eq. (7) is defined as the local gradient of the output layer, expressed as $\delta_{k}$.

$$
\begin{gathered}
\delta_{k}=\left(-\frac{\partial_{\varepsilon}}{\partial c_{k}^{K}}\right)=\left(-\frac{\partial_{\varepsilon}}{\partial O_{k}^{K}}\right) \times\left(-\frac{\partial o_{k}^{K}}{\partial v_{k}^{K}}\right) \\
=\left(d_{k}-O_{k}^{K}\right) \times\left(\frac{\partial_{\varphi}\left(v_{k}^{K}\right)}{\partial v_{k}^{K}}\right) \\
=\left(d_{k}-O_{k}^{K}\right) \times \varphi^{\prime}\left(v_{k}^{K}\right) \\
=\frac{1}{2}\left(d_{k}-O_{k}^{K}\right)\left[1-\left(O_{k}^{K}\right)^{2}\right]
\end{gathered}
$$

Combining Eqs. (6) and (9), the update formula for the weights of neurons in the output layer is obtained:

$w_{k j}^{K J}(n+1)=w_{k j}^{K J}(n)+\eta_{k}\left\{\frac{1}{2}\left(d_{k}-o_{k}^{K}\right)\left[1-\left(o_{k}^{K}\right)^{2}\right]\right\} o_{j}^{J}$

The BP algorithm also updates the hidden layer weights in a gradient descent manner. In each iteration, the correction amount of the hidden layer weight is:

$$
\begin{gathered}
\Delta w_{j i}^{J I}=\eta_{j}\left(-\frac{\partial \varepsilon}{\partial w_{j i}^{J l}}\right) \\
=\eta_{j}\left(-\frac{\partial \varepsilon}{\partial v_{j}^{J}}\right) \times \eta_{j}\left(-\frac{\partial v_{j}^{J}}{\partial w_{j i}^{J l}}\right) \\
\triangleq \eta_{j} \delta_{j} \times\left(-\frac{\partial v_{j}^{J}}{\partial w_{j i}^{J l}}\right)
\end{gathered}
$$

where $\eta_{j}$ is the learning rate of the $j$-th neuron in the hidden layer, and $\delta_{j}$ is the local gradient of the hidden layer.
Because of $v_{j}^{J}=\sum_{i=1}^{I} w_{j i}^{J I} o_{j}^{J}+b_{j}^{J}$, there is $\left(-\frac{\partial v_{j}^{J}}{\partial w_{j i}^{J l}}\right)=o_{i}^{I}$. The local gradient $\delta_{j}$ of the hidden layer satisfies:

$$
\begin{gathered}
\delta_{j}=\left(-\frac{\partial \varepsilon}{\partial v_{j}^{J}}\right)=-\left(\frac{\partial \varepsilon}{\partial O_{k}^{K}}\right) \times\left(\frac{\partial O_{k}^{K}}{\partial v_{k}^{K}}\right) \times\left(\frac{\partial v_{k}^{K}}{\partial O_{j}^{J}}\right) \times\left(\frac{\partial O_{j}^{J}}{\partial v_{j}^{J}}\right) \\
=-\sum_{k=2}^{K}\left(\frac{\partial \varepsilon}{\partial O_{k}^{K}}\right) \times \varphi^{\prime}\left(v_{k}^{K}\right) \times\left(\frac{a v_{k}^{K}}{\partial O_{j}^{J}}\right) \varphi^{\prime}\left(v_{j}^{J}\right) \\
=\sum_{k=1}^{K}\left(d_{k}-O_{k}^{K}\right) \times \frac{1}{2}\left[1-\left(O_{k}^{K}\right)^{2}\right] \times w_{k j}^{K J} \times \frac{1}{2}\left[1-\left(O_{j}^{J}\right)^{2}\right] \\
=\left(\sum_{k=1}^{K} \delta_{k} w_{k j}^{K J}\right) \times \frac{1}{2}\left[1-\left(O_{j}^{J}\right)^{2}\right]
\end{gathered}
$$

From Eq. (12), the local gradient $\delta_{k}$ of the output layer has an effect on the local gradient $\delta_{j}$ of the hidden layer, that is, the error function flows to the hidden layer through the output layer. Therefore, the update formula for the weights of hidden layer neurons is as follows:

$$
\begin{gathered}
w_{j i}^{J I}(n+1)=w_{j i}^{J I}(n)+\Delta w_{j i}^{J I}=w_{j i}^{J I}(n)+\eta_{j} \delta_{j} o_{i}^{I} \\
=w_{j i}^{J I}(n)+\eta_{j}\left(\sum_{k=1}^{K} \delta_{k} w_{k j}^{K J}\right) \times \frac{1}{2} \delta_{k}\left[1-\left(o_{j}^{J}\right)^{2}\right] o_{i}^{I}
\end{gathered}
$$

In the training process of the neural network, the error between the actual output signal and the expected signal constitutes a cost function, which is the driving force for the continuous training and adjustment of the network. Therefore, in the neural network model shown in Fig. 2, the input signal is transmitted layer by layer from left to right, and the network modifies the weights layer by layer from right to left through the BP learning algorithm.

\subsection{Multi Step Prediction Algorithm Based on Cascaded Neural Network (CFN)}

The structure of CFN is similar to FFN, but the input signal in the network is connected to each hidden layer behind it through a weight matrix. The structure of CFN is shown in Fig. 3.

In CFN, all hidden layers except the first hidden layer contain two weight matrices $\boldsymbol{W}_{\mathbf{1}}$ and $\boldsymbol{W}_{\mathbf{2}}$, which are used to control the output signal of the upper layer and the input signal of the network, respectively. This structure can give more degrees of freedom to the training process, thereby improving the non-linear mapping ability of the network. Therefore, the cognitive base station can perceive the behavior of authorized users in the next few time slots, and perform access control or spectrum switching for cognitive users in time to minimize interference to authorized users. For CFN, the BP learning algorithm shown in Fig. 4 is used for training to optimize the weight matrix $\boldsymbol{W}_{\mathbf{1}}, \boldsymbol{W}_{\mathbf{2}}$ and bias matrix $\boldsymbol{b}_{\mathbf{i}}$ of the network. Its goal is to make the 
Fig. 3 Structure of cascade neural network

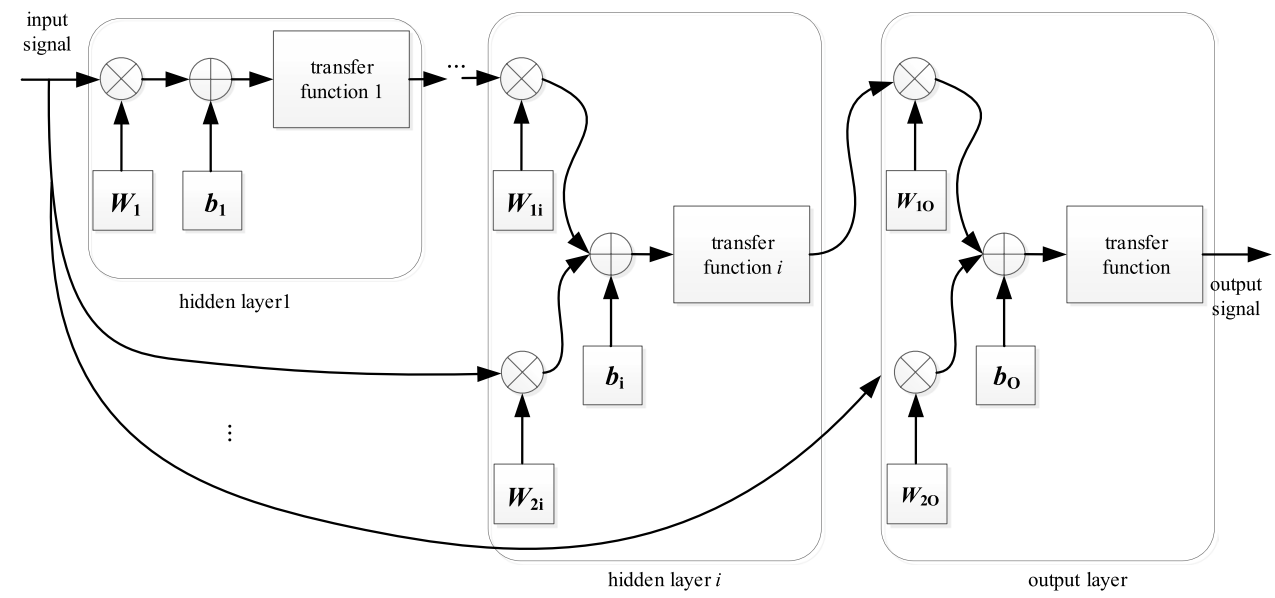

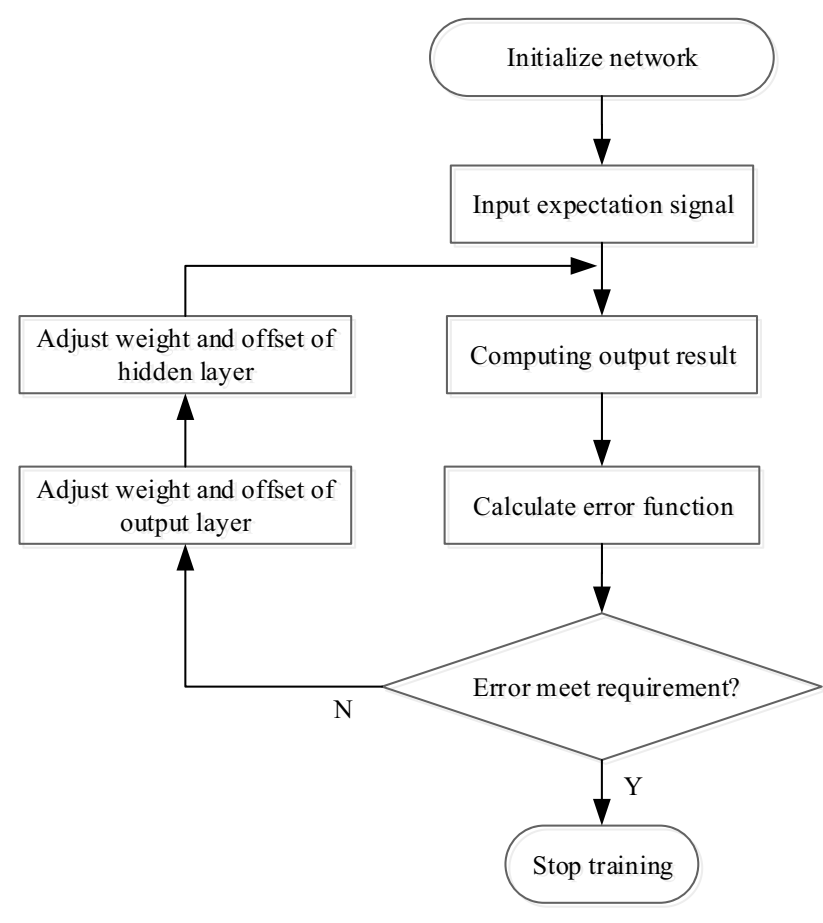

Fig. 4 Flow chart of BP learning algorithm

actual output of the network closest to the expected output in the sense of the smallest mean square error.

However, the change of the CFN structure increases the complexity of the training process, because each iteration of BP training needs to modify more weight matrices. In addition, if too many training samples are provided to the network, it will also lead to overfitting. The multi-step prediction algorithm based on CFN makes use of the strong nonlinear mapping ability of CFN to predict the authorized user behavior in the next several sensing time slots. CFN also uses $\left[S_{t-\tau+1}, S_{t-\tau+2}, \ldots S_{t-1}, S_{t}\right]^{T}$ as the network input. The difference from FFN is that the network outputs the predicted value $\left[\widehat{S}_{t+1}, \widehat{S}_{t+2}, \ldots \widehat{S}_{t+M}\right]^{T}$ of user behavior in the $M(M \geq 2)$ sensing slots in the future. In addition, the expected value of the network during the training process is also a column vector $\left[S_{t+1}, S_{t+2}, \ldots \widehat{S}_{t+M}\right]^{T}$ of length $M$.

With the prediction of the neural network, the behavior $s_{t+1}(1 \leq i \leq M)$ of authorized users in each sensing slot in the future will be predicted $M$ times. The cognitive base station can combine the prediction results of $M$ times to schedule cognitive users on this basis. However, with the increase of the time interval between the target sensing time slot and the current time window, the correlation between $s_{t+1}(1 \leq i \leq M)$ in the future sensing time slot and historical data in the time window is decreasing. That is, as the number of sensing time slots $i$ increases, the deviation between the estimated result $\widehat{S}_{t+1}(1 \leq i \leq M)$ and the actual result $s_{t+1}(1 \leq i \leq M)$ of the authorized user will increase.

In order to reduce the interference to authorized users, the cognitive base station in the network adopts the hard decision mode of "or" combination. For the $M$ prediction results of the behavior of the authorized user in the next perception time slot, if there is an authorized user occupying the subband, the cognitive base station considers that the authorized user will occupy the sub-band.

\section{Simulation Results and Analysis}

FFN and CFN use a hidden layer, the number of neurons in the hidden layer is 10 , and the transfer function of the hidden layer is $\varphi(\bullet)$. The number of neurons in the input layer and output layer is the same as the length $\tau$ of training time window. FFN and CFN use the same training samples for training, and the simulation results are shown in Fig. 5.

Figure 5 shows that the convergence speed of CFN is faster than FFN. In addition, after 40 iterations, the mean 


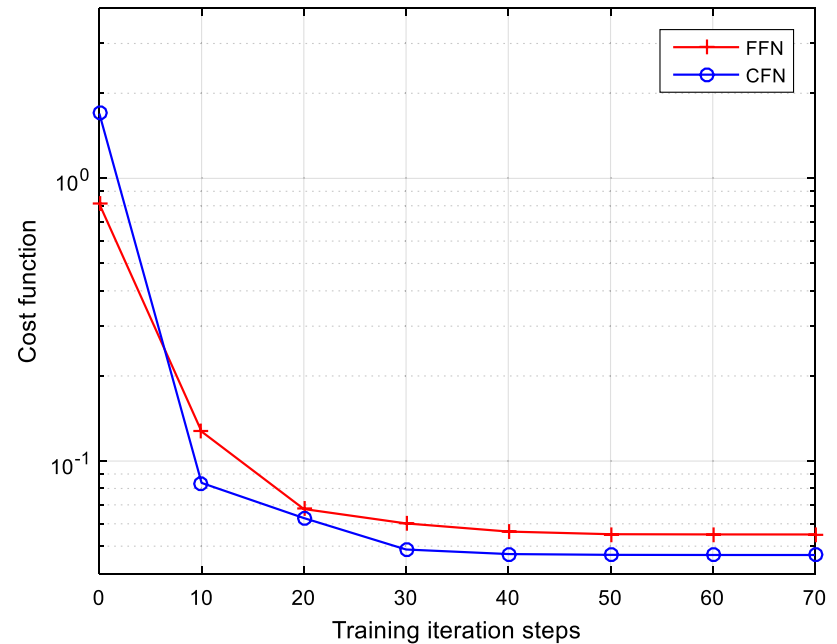

Fig. 5 Training results of artificial neural networks

square error of CFN is less than FFN, but all remains unchanged. Therefore, CFN has stronger learning and generalization capabilities than FFN, but due to the high complexity of the network structure, relatively long training time is required.

For user behavior prediction algorithms based on FFN and CFN, the prediction order will have a great impact on the predicted conflict probability. A larger prediction order increases the number of neurons in the input layer and increases the complexity of the network, thereby weakening the training effect of the network. On the other hand, a smaller prediction order cannot satisfy the non-linear mapping relationship from input to output. Simulate the exponential ON/OFF model of authorized users and compare the conflict probability of the algorithm under different prediction orders. The parameters used are shown in Table 1.

The user behavior prediction algorithms studied in this paper all use Monte Carlo simulation for 10,000 times. The curve of the algorithm's spectrum conflict probability with the network prediction order is shown in Fig. 6.

When the prediction order is greater than 4 , the conflict probability of the multi-step prediction algorithm based on CFN is significantly lower than that one step prediction algorithm based on FFN. When the prediction order is set to 2 or 3 , the conflict probability is almost the same. On the other hand, the conflict probability of the two algorithms is the smallest when the prediction order is 5 . Therefore, in the authorized user exponential ON/OFF model studied in this paper, two prediction algorithms based on neural networks set the prediction order to 5 .

Set service parameter $e_{1} \in[2,10]$ and $e_{1}=5$, and other simulation parameters are shown in Table 1 . The error prediction probability in Fig. 7 shows that with the increase of $e_{1}$, the error prediction probability $P_{\text {overall }}^{e}$ of the three

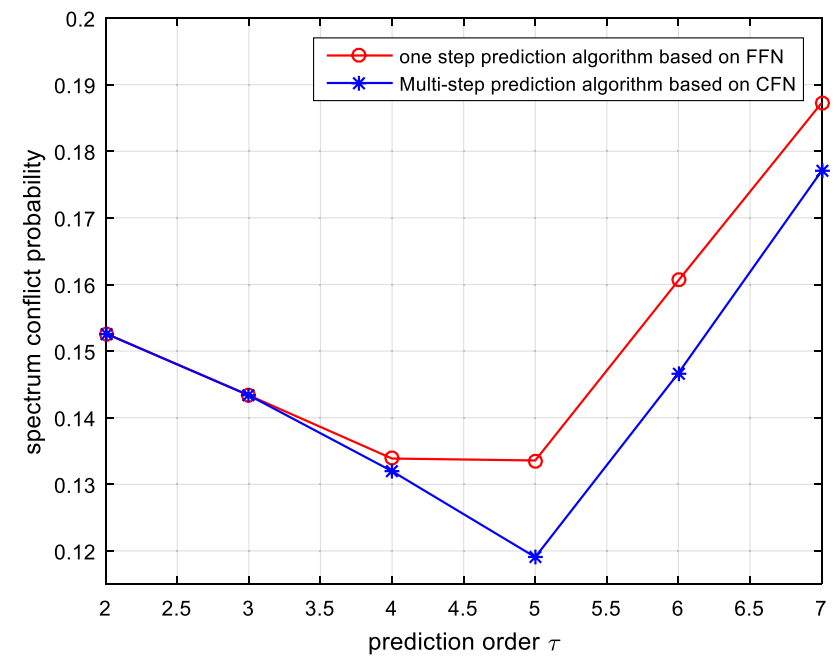

Fig. 6 spectrum conflict probability with prediction order.

Table 1 prediction algorithm simulation parameters.

\begin{tabular}{ll}
\hline Parameter name & value \\
\hline parameter $e_{1}$ & 2 \\
parameter $e_{2}$ & 5 \\
Sensing slot length $\Delta t$ & $20 \mathrm{~ms}$ \\
Number of hidden layers & 2 \\
Number of neutrons in hidden layer & 10 \\
Prediction steps $M$ of CFN based algorithm & 2 \\
Number of training sample & 2000 \\
Maximum iterative steps of BP algorithm & 500 \\
\hline
\end{tabular}

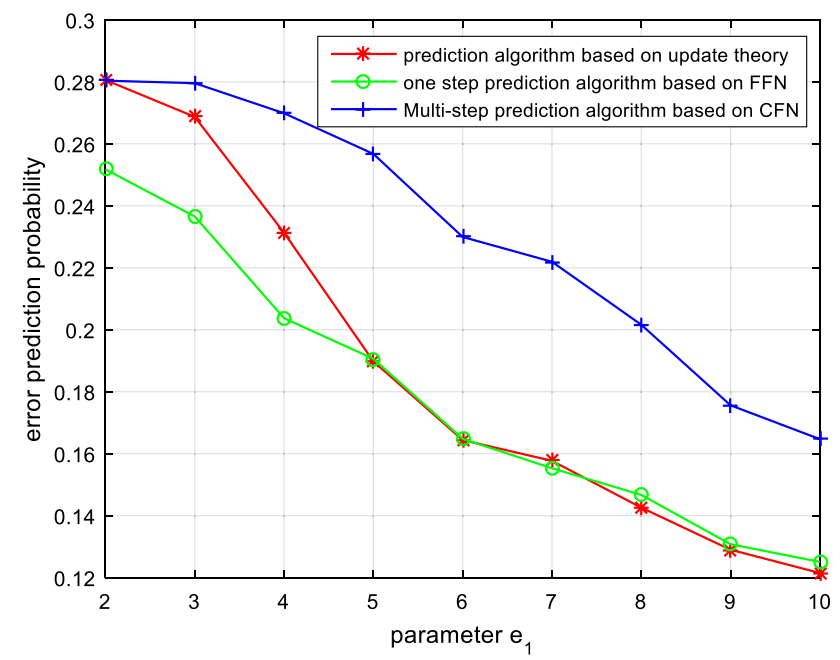

Fig. 7 Error prediction probability with business parameter $e_{1}$ 
prediction algorithms gradually decreases. Because the increase in $e_{1}$ means that the average time for authorized users to stay in the $\mathrm{ON}$ state increases, while $e_{2}$ remains the same, resulting in a decrease in the frequency of authorized users to switch between ON/OFF states. Therefore, the correlation of the behavior of authorized users in adjacent sensing time slots is increased, making it easier for the cognitive base station to predict its behavior.

In addition, it can be found that the error prediction probability of the Multi-step prediction algorithm based on CFN is higher than the other two algorithms. This is because the algorithm adopts the "or" combination hard decision method when judging the results of $M$ predictions. Although this method can well avoid the spectrum conflict between cognitive users and authorized users, some spectral holes are also missed. On the other hand, when $e_{1}$ is less than 5 , the error prediction probability of one step prediction algorithm based on FFN is lower than that based on update theory. When e is greater than 5 , the performance of the two algorithms is almost the same. Therefore, compared with the prediction algorithm based on the update theory, the algorithm based on the neural network has better prediction accuracy in the environment where the status of authorized users changes rapidly.

Figure 8 shows the conflict probability of the algorithm under different authorized user service parameters. Comparing Figs. 7 and 8, the conflict probability in the prediction process is lower than the error prediction probability. This is because there are two types of mispredictions: spectrum conflicts and cognitive base stations mistakenly believe that spectrum holes are occupied. The former measures the interference risk of cognitive users to authorized users from the perspective of authorized users, and the latter measures the

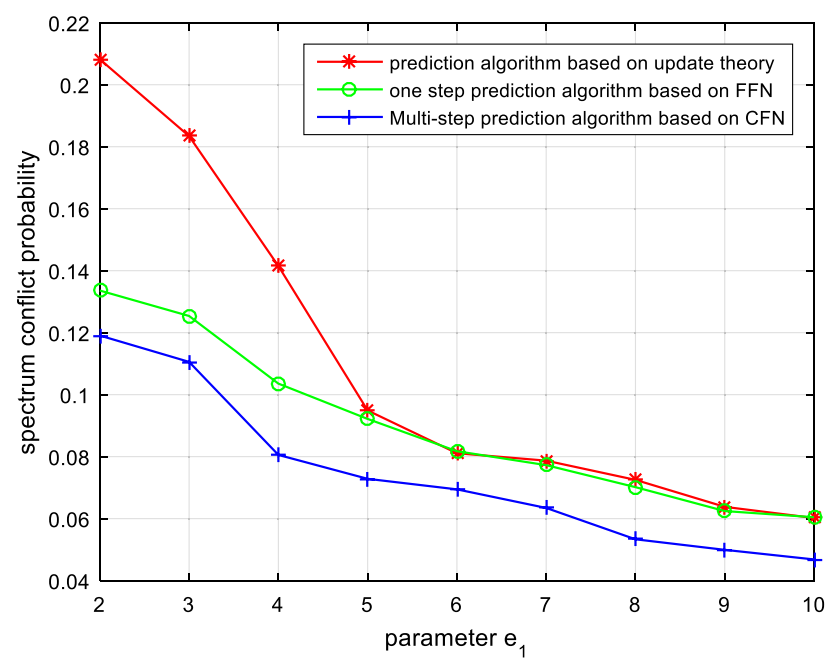

Fig. 8 Spectrum conflict probability with business parameter $e_{1}$ utilization of spectrum resources from the perspective of cognitive users.

The conflict probability of the two prediction algorithms based on neural networks is lower than that of the traditional prediction algorithms based on update theory, indicating that it is feasible to use artificial neural networks. Compared with the prediction method based on the update theory, the neural network avoids the complicated description of the characteristics of authorized users, and uses the powerful learning ability and nonlinear mapping ability to effectively describe the relationship between input and output. Therefore, the prediction method based on neural network can realize prediction by learning and extracting historical information from the spectrum database without knowing the service parameters of authorized users. On the other hand, the cognitive base station adopts the "offline" mode to predict the trained network, only a small amount of addition and multiplication operations are needed to realize the prediction function, and the prediction speed of the two algorithms is relatively fast.

Compared with the one step prediction algorithm based on FFN, the multi-step prediction algorithm based on CFN can effectively reduce the probability of spectrum conflict in the network. In addition, the multi-step prediction algorithm based on CFN further reduces the probability of conflict by increasing the number of prediction steps. However, it also increases the probability of prediction errors, leading to a decrease in spectrum utilization in cognitive wireless networks. Therefore, in practical applications, cognitive base stations need to balance the utilization of spectrum resources and interference to authorized users.

\section{Conclusion}

This article discusses the behavior prediction algorithm of authorized users in satellite cognitive wireless networks. First, it analyzes the exponential ON/OFF model of user behavior prediction in satellite cognitive wireless network and the learning mechanism of artificial neural network. Then study the traditional prediction algorithm based on update theory and FFN, and propose a prediction algorithm based on CFN. The algorithm uses CFN's powerful learning ability and non-linear mapping ability to realize multi-step prediction of authorized user behavior, judge the results of multiple predictions of user behavior in a certain sensing time slot, and reduce the spectrum conflict between cognitive users and authorized users. Finally, the simulation results show that the CFN-based multi-step prediction algorithm proposed in this paper can effectively reduce the probability of spectrum conflicts in cognitive wireless networks. Cognitive base stations using predictive algorithms can effectively reduce the interference of cognitive users to 
authorized users through reasonable access management and scheduling methods.

Acknowledgements The paper is sponsored by National Natural Science Foundation of China (No. 62071146), Projects of Science and Technology on Communication Networks Laboratory, Grant/Award Number: 6142104190113.

Open Access This article is licensed under a Creative Commons Attribution 4.0 International License, which permits use, sharing, adaptation, distribution and reproduction in any medium or format, as long as you give appropriate credit to the original author(s) and the source, provide a link to the Creative Commons licence, and indicate if changes were made. The images or other third party material in this article are included in the article's Creative Commons licence, unless indicated otherwise in a credit line to the material. If material is not included in the article's Creative Commons licence and your intended use is not permitted by statutory regulation or exceeds the permitted use, you will need to obtain permission directly from the copyright holder. To view a copy of this licence, visit http://creativecommons.org/licenses/by/4.0/.

\section{References}

1. Liu X, Zhai XB, Lu W, Wu C (2021) QoS-Guarantee Resource Allocation for Multibeam Satellite Industrial Internet of Things With NOMA. IEEE Trans Industr Inf 17(3):2052-2061. https:// doi.org/10.1109/TII.2019.2951728

2. Vassaki S, Poulakis MI, Panagopoulos AD et al (2013) Power Allocation in Cognitive Satellite Terrestrial Networks with QoS Constraints [J]. IEEE Commun Lett 17(7):1344-1347

3. Liu X, Zhang X, Jia M et al (2018) 5G-based green broadband communication system design with simultaneous wireless information and power transfer[J]. Physical Communication 28:130-137

4. Sharma SK, Chatzinotas S, Ottersten B. Exploiting polarization for spectrum sensing in cognitive SatComs [C].7th International Conference on CROWNCOM: Stockholm, Sweden, June, 2012: 36-41.

5. Li F, Lam K, Liu X et al (2018) Joint Pricing and Power Allocation for Multibeam Satellite Systems With Dynamic Game Model. IEEE Trans Veh Technol 67(3):2398-2408. https://doi.org/10. 1109/TVT.2017.2771770

6. Morlet C. Software Radio in Space Segment: Applications, Technologies, Reconfiguration Management and Architectures [C]. International Workshop on Satellite and Space Communications. 2006: 269-270.

7. Tang W, Thompson P, Evans B. Frequency sharing between satellite and terrestrial systems in the Ka band: A database approach
[C]. IEEE International Conference on Communications, 2015: 867-872.

8. Liu QL, Ren GC, Tao YR (2007) DARPA Next Generation Program [J]. Journal of Military Communications Technology 28(3):77-81

9. Suffritti R, Corazza G E, Guidotti A, et al. Cognitive hybrid satellite-terrestrial systems.[C]. CogART 2011, International Conference on Cognitive Radio and Advanced Spectrum Management, October 26-29, Barcelona, Spain, 2011: 1-5.

10. Shree Krishna Sharma, Symeon Chatzinotas and Björn Ottersten. Cognitive beamhopping for spectral coexistence of multibeam satellites [J]. International Journal of Satellite Communications and Networking, 2014: 1-22.

11. Park U, Kim H W, Oh D S, et al. "Performance Analysis of Dynamic Resource Allocation for Interference Mitigation in Integrated Satellite and Terrestrial Systems"[C]//International Conference on Next Generation Mobile Applications, Services and Technologies. IEEE, 2016: 217-221.

12. Liu X, Zhang X (2019) Rate and Energy Efficiency Improvements for 5G-Based IoT With Simultaneous Transfer. IEEE Internet Things J 6(4):5971-5980. https://doi.org/10.1109/JIOT.2018. 2863267

13. Zhu X, Jiang C, Feng W, et al. Resource allocation in spectrumsharing Cloud Based Integrated Terrestrial-Satellite Network[C]// International conference on wireless communications and mobile computing, 2017: 334-339.

14. Zheng H (2007) Proactive Channel Access in Dynamic Spectrum Networks $[\mathrm{J}]$. Chinese Journal of Sensors \& Actuators 20(2):393-397

15. Lo Presti F, Zhang ZL, Kurose J et al (1999) Source time scale and optimal buffer/bandwidth tradeoff for heterogeneous regulated traffic in a network node [J]. IEEE/ACM Trans Networking 7(4):490-501

16. Marinho J, Monteiro E (2012) Cognitive radio: Survey on communication protocols, spectrum decision issues, and future research directions [J]. Wireless Netw 18(2):1-18

17. Yarkan S, Arslan H. Binary Time Series Approach to Spectrum Prediction for Cognitive Radio [C]. IEEE Vehicular Technology Conference. 2007: 1563-1567.

18. Cox DR (1962) Renewal Theory [J]. Encyclopedia of Statistical Sciences 4(01):281-302

19. Tumuluru V K, Wang P, Niyato D. A Neural Network Based Spectrum Prediction Scheme for Cognitive Radio [J]

Publisher's Note Springer Nature remains neutral with regard to jurisdictional claims in published maps and institutional affiliations. 